\title{
Search for a permanent electric dipole moment using liquid ${ }^{129} \mathrm{Xe}$ PI: Michael Romalis Final Technical Report
}

Search for an electric dipole moment is one of the best motivated low-energy approaches for investigating physics beyond the Standard Model. Our experimental effort is focused on improving the limit on EDM in liquid ${ }^{129} \mathrm{Xe}$ to put constraints on nuclear $\mathrm{CP}$-violating interactions.

High nuclear spin density and high electrical breakdown strength make ${ }^{129} \mathrm{Xe}$ a promising medium for EDM searches. At the time the project started, the transverse nuclear spin relaxation time $\mathrm{T}_{2}$ of ${ }^{129} \mathrm{Xe}$ was unknown. We made measurements of $\mathrm{T}_{2}$ using NMR spin-echo techniques and found that it is exceeds $1300 \mathrm{sec}$, the longest relaxation time ever measured in a liquid [1]. We also began to investigate non-linear dipolar interaction effects in a high-density spinpolarized liquid Xe.

In the second iteration of the experiment we setup a high-Tc SQUID system in magnetic shields and performed detailed studies of Xe spin precession. We developed a model for nonlinear dipolar interactions and found that for one set of conditions non-linear interactions can delay spin dephasing due to magnetic field gradients, while for another set of conditions they can lead to exponential amplification of the spin precession signals [2]. Our experimental data were in good quantitative agreement with predictions of the model.

We also developed a series of numerical simulations to understand various imperfections in the system and made detailed experimental measurements to confirm these numerical predictions [3]. We demonstrated that non-linear interactions can amplify small precession signals and achieved an amplification factor of 10 [4]. This general phenomenon can be used in other precision measurements with non-linear interactions.

We also explored practical applications of the liquid Xe system that we developed. We demonstrated that by mixing Xe with organic liquids, such as cyclopentane, one can enhance the proton spin polarization by a factor of $10^{6}$ [5]. We have used this technique to perform the first measurement of the scalar J-coupling between nuclear spins in van-der-Waals molecules, something that has never been observed before.

More recently, we constructed a liquid-He apparatus to acquire Xe spin precession data using a low-Tc SQUID and achieved a signal-to-noise ratio of $10^{6}$. We are currently investigating factors affecting the stability of Xe spin precession signals in this system using a superconducting magnetic shield and a persistent current magnetic field coil.

[1] M. V. Romalis and M. P. Ledbetter. "Transverse Spin Relaxation in Liquid ${ }^{129} \mathrm{Xe}$ in the presence of Large Dipolar Fields." Phys. Rev. Lett. 87, 67601 (2001).

[2] M. P. Ledbetter and M. V. Romalis. "Nonlinear Effects from Dipolar Interactions in Hyperpolarized Liquid ${ }^{129}$ Xe." Phys. Rev. Lett. 89, 287601 (2002).

[3] M. P. Ledbetter, I. M. Savukov, L.-S. Bouchard, and M. V. Romalis. "Numerical and experimental studies of long-range magnetic dipolar interactions." J. Chem. Phys. 121, 1454 (2004).

[4] M. P. Ledbetter, I. M. Savukov, and M. V. Romalis. "Nonlinear Amplification of Small Spin Precession Using Long-Range Dipolar Interactions." Phys. Rev. Lett. 94, 060801 (2005).

[5] J. J. Heckman, M. P. Ledbetter, and M. V. Romalis, Enhancement of SQUID-Detected NMR Signals with Hyperpolarized Liquid ${ }^{129} \mathrm{Xe}$ in a $1 \mu \mathrm{T}$ Magnetic Field, Phys. Rev. Lett. 91, 067601 (2003). 